\title{
Lifetime Estimation for the Power Semiconductors Considering Mission Profiles in Wind Power Converter
}

\author{
Ke Ma, Marco Liserre, Frede Blaabjerg \\ Department of Energy Technology \\ Aalborg University, Aalborg 9220, Denmark \\ kema@et.aau.dk, mli@et.aau.dk, fbl@et.aau.dk
}

\begin{abstract}
As a key component in the wind turbine system, power electronic converter and its power semiconductors suffer from adverse power loadings related to environment, and are proven to have certain failure rates. Therefore, correct lifetime estimation of wind power converter is crucial for the reliability improvement and also for cost reduction of wind power technology. Unfortunately, the existing lifetime estimation methods for the power electronic converter are not yet suitable in the wind power application, because the comprehensive mission profiles are not well specified and included. Consequently, a relative more advanced approach is proposed in this paper, which is based on the loading and strength analysis of devices and takes into account different time constants of the thermal behaviors in power converter. With the established methods for loading profile generation and lifetime estimation, more detailed information for the reliability performance of wind power converter can be obtained.
\end{abstract}

\section{INTRODUCTION}

The fast growth in the total installation and individual capacity makes the failures of wind turbines more critical for the power system stability and also more costly to repair [1][3]. Former field feedbacks have shown that the power electronics tend to have higher failure rate than the other parts in the wind turbine system [4],[5]. As a result, correctly estimating the reliability performance of the wind power converter is crucial, not only for lifetime extension, but also for the cost reduction of the wind power technology [6], [7].

The reliability research in power electronics has been carried out for decades. As the state-of-the-art agreement, the reliability engineering in power electronics is now moving from a solely statistical approach that has been proven to be unsatisfactory in the automotive industry, to a more physics based approach which involves not only the statistics but also the root cause behind the failures [8]-[14]. In this approach the correct mapping of loading profile which can trigger the failures of components (the stress analysis), as well as the lifetime modeling which investigates how much loading the components can withstand (the strength models), are two of the most important factors for lifetime or reliability estimation, as illustrated in Fig. 1.

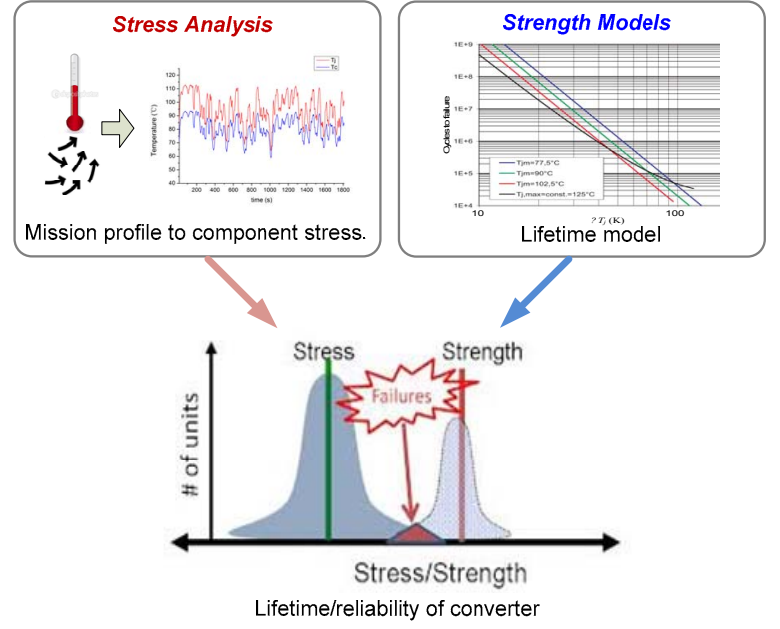

Fig. 1. Physics-based life time estimation for power electronic components.

It has been revealed that the thermal cycling (i.e. temperature swings inside or outside the devices) is one of the most critical failure causes in power electronics system [11]-[14]. The thermal cycling among different contacting materials with various Coefficients of Thermal Expansion (CTE) will cause the crack or deformation in the contacting points, thus leading to the failures of the devices. Many manufacturers of power electronic devices such as power semiconductors or capacitors have developed their reliability models, which normally are based on accelerating or aging tests, and are able to transfer certain thermal cycling of components into the corresponding lifetime information [15]-[18]. However, correctly mapping a mission profile of converter into the specific loading profile of power electronic components is still a challenging task: For example in the wind power system, various dynamical changes of wind speeds or ambient temperature at time constants ranging from seconds to months, together with the corresponding control behaviors of turbine, generator and converter, all lead to complicated loading profiles of the devices that are difficult to be handled when doing reliability calculation.

Some simplified methods for the loading profile mapping have been used in traction and electric vehicle applications - 
they typically focus on a selected and tough operating condition of the converters in a very short term, and then the longer term loading profiles are generated by simply repeating the results acquired in short term [16], [19]-[21]. However this approach may lead to significant deviation from reality in applications with more complicated mission profiles. For example in the wind power converter the thermal loading of power devices does not periodically repeat but randomly changes with the wind speeds and ambient temperature. Moreover, these existing lifetime estimations can just acquire the general life time of devices, while the life time distribution - which indicates the failure contribution by different loading conditions as well as failure mechanisms, would be more useful for the design and improvement of converter reliability.

In this paper, a more advanced approach for thermal profile mapping as well as lifetime estimation of wind power converter is proposed. It is based on the failure mechanism of power semiconductor devices, i.e. thermal stress generation and life time modeling, and a more complete mission profile of wind power converter are processed considering different time constants. In the end some possibilities as well as limits of the proposed method are also discussed.

\section{BASIC IDEA AND MISSION PROFILE FOR STUDY}

As mentioned before, correct transforming the mission profile of wind power converter into the corresponding loading profile of the power devices is not an easy task: First, many factors which have influence on the thermal loading of devices should be taken into account, like the wind speed and ambient variations, behaviors of mechanical parts, behaviors of electrical parts, and also grid conditions, etc. These considerations may involve multi-disciplinary models with quite different time constants, and therefore it is difficult to evaluate these models together at the same time step in order to acquire the interested thermal behaviors. Second, in the case of long term analysis e.g. one year operation which is necessary for lifetime estimation, large amount of loading data may be generated, which is difficult to be handled if considering too many details of the system; On the contrary, if too rough models and longer time step are used, the generated loading profile may not contain enough thermal dynamics and the lifetime information may significantly deviate from the reality. Therefore, it is important first to develop a way to properly extract and sort the thermal loading in wind power converter for the sake of lifetime estimation.

Inspired by the approach used in photography, where lenses with different focal length are widely used to acquire the images with different sizes and details, the thermal behaviors of the power device in wind power converter can be also focused at different "focal length", which in this case is represented by time constants or time step. According to the main causes of loading change in a wind power converter, the thermal behaviors of power electronic components can be generally classified into three time constants: long term, medium term, and short term, as indicated in Fig. 2. It is noted that in each of the time constant the interested loading behaviors, time step and model details are different, thereby the thermal loading of components can be more efficiently generated. After the loading profiles in each of the time domain are acquired, the corresponding lifetime performance can be estimated respectively, and then be combined together according to the Miner's rule [22].

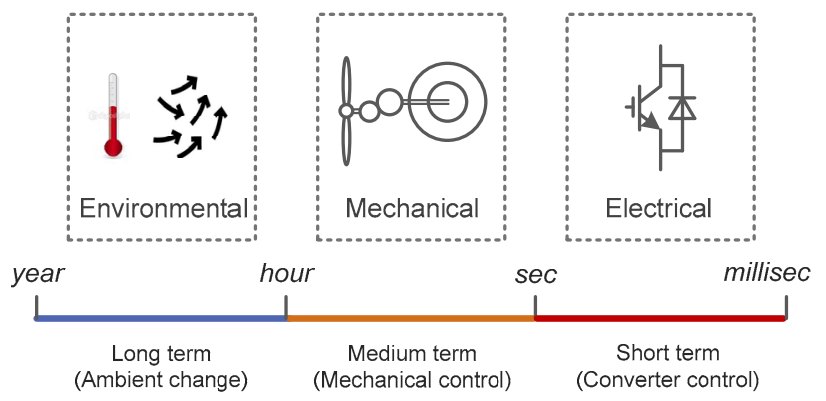

Fig. 2. Thermal cycles of power semiconductors in a wind power converter with three different time constants.

A typical wind condition and wind turbine system has to be settled first as a study case. As shown in Fig. 3, a 1 year wind speed and ambient temperature profile is used with 3 hours averaged at $80 \mathrm{~m}$ hub height, which were designated for the wind farm located near Thyborøn, Denmark with latitude $56.71^{\circ}$ and longitude $8.20^{\circ}$. The chosen hub wind speed belongs to the wind class IEC I with average wind speed of $8.5-10 \mathrm{~m} / \mathrm{s}$ [23], [24] and a $2.0 \mathrm{MW}$ wind turbine $\mathrm{V} 80[25]$ is chosen to fit the given wind condition.
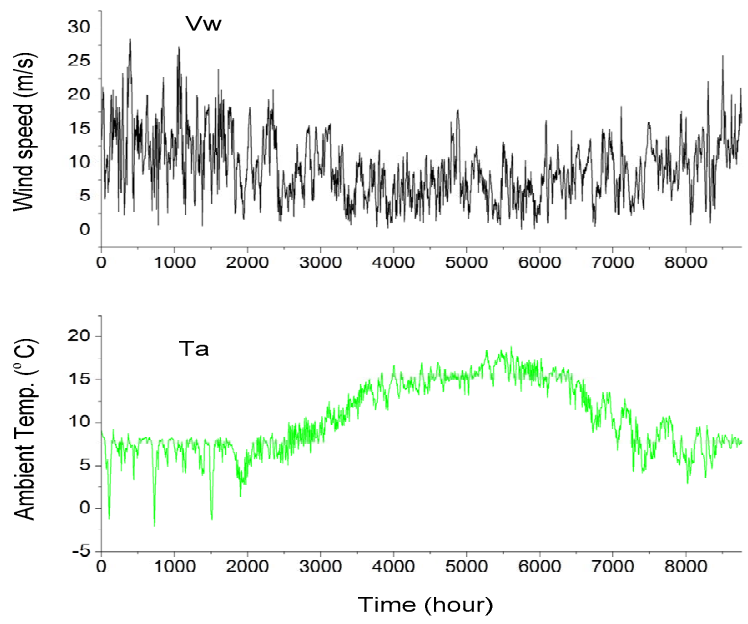

Fig. 3. One year mission profile of wind speed and ambient temperature from a wind farm (3-hour averaged).

In respect to the wind power converter, the most adopted two-level back-to-back voltage source converter topology is chosen, as shown in Fig. 4. Only the grid side converter is 
chosen as a case study, whose parameters are basically designed according to Table I, which is a state-of-the-art configuration for the two level wind power converter [1], [3]. The generator side converter can share the similar approach for analysis.

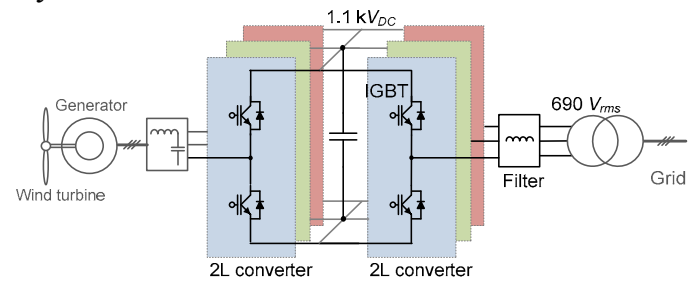

Fig. 4. Wind power converter for lifetime estimation.

Table I. Parameters of converter in Fig. 4.

\begin{tabular}{|l|c|}
\hline Rated output active power $P_{o}$ & $2 \mathrm{MW}$ \\
\hline DC bus voltage $V_{d c}$ & $1.1 \mathrm{kV} \mathrm{DC}$ \\
\hline${ }^{*}$ Rated primary side voltage $V_{p}$ & $690 \mathrm{~V} \mathrm{rms}$ \\
\hline Rated load current $I_{\text {load }}$ & $1.93 \mathrm{kA} \mathrm{rms}$ \\
\hline Fundamental frequency $f_{o}$ & $50 \mathrm{~Hz}$ \\
\hline Switching frequency $f_{c}$ & $1950 \mathrm{~Hz}$ \\
\hline Filter inductance $L_{f}$ & $132 \mu \mathrm{H}(0.2$ p.u. $)$ \\
\hline
\end{tabular}

* Line-to-line voltage in the primary windings of transformer.

\section{LONG TERM LOADING PROFILES AND LIFETIME ESTIMATION}

As indicated in section II, the life time estimation of wind power converter is going to be conducted under different time constants. In this section the condition with long time constant will be analyzed. It is noted that this group of models and estimations only focus on the long term thermal behaviors and corresponding lifetime caused by the environmental disturbances, e.g. the variation of wind speeds or the ambient temperatures in a few days or months. Therefore, simplified models and large time step are generally used.

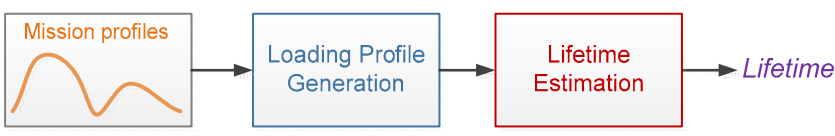

Time span : 1 year, Step: 3 hours

Fig. 5. Flow-chart for life time estimation of power devices caused by long term thermal cycles.

As shown in Fig. 5, the flow for the life time estimation under long term time constant is straightforward: the wind profiles in Fig. 3 is directly fed into a series of wind turbine models in order to generate the corresponding thermal loading of the power devices. And then the acquired thermal loading is processed for life time estimation. The analysis in this section is conducted with a time span of 1 year and step of 3 hours, which is synchronized with the wind speed and ambient temperature profiles given in Fig. 3.

\section{A. Long term loading profile generation}

In Fig. 6, a diagram for long term loading profile generation is indicated. It can be seen that multi-disciplinary models like the wind turbine, generator, converter, as well as loss and thermal characteristics of the power devices are all included in order to map the mission profile of the wind turbines into the thermal loading of power semiconductors.

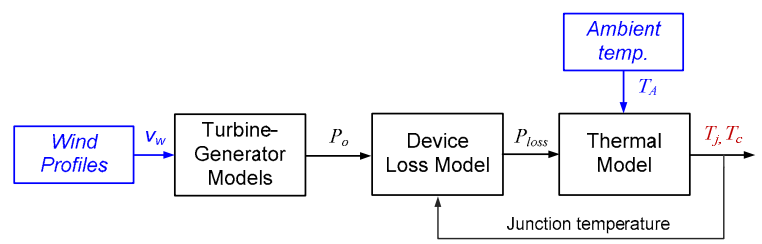

Fig. 6. Multi-domain models used for thermal profile generation of power devices.

Because the wind speed is sampled at 3 hours and only long term thermal behaviors are focused, the inertia effects of the wind turbine and generator which normally range in the time constant of seconds to minutes can be ignored. The output power of wind turbine can be looked up from the power curve provided by the manufacturer [25] and can be directly used as the delivered power of converter.

Similarly, because the response time of converter power and corresponding losses is much smaller than the interested time constant, the loss on the power semiconductor can be directly acquired from lookup table in order to accelerate the analyzing speed. The IGBT module from ABB 5SNA $2400 \mathrm{E} 170305\left(2.4 \mathrm{kA} / 1.1 \mathrm{kV} / 150^{\circ} \mathrm{C}\right)$ are chosen as power semiconductor devices, which in this paper have maximum junction temperature at $115^{\circ} \mathrm{C}$, when the liquid temperature of water-cooled heat sink is at $40^{\circ} \mathrm{C}$. In order to enable the temperature dependency of the device losses [26], [27], a 3dimension lookup table is established. As shown in Fig. 7, the losses consumed by the power devices are decided by the input power of converter as well as device junction temperature. For the sake of accuracy, each of the point in the lookup table is simulated in a detailed circuit model with complete switching behaviors of the power devices, and the conduction loss, switching loss and diode reverse recovery loss are all taken into account [26].

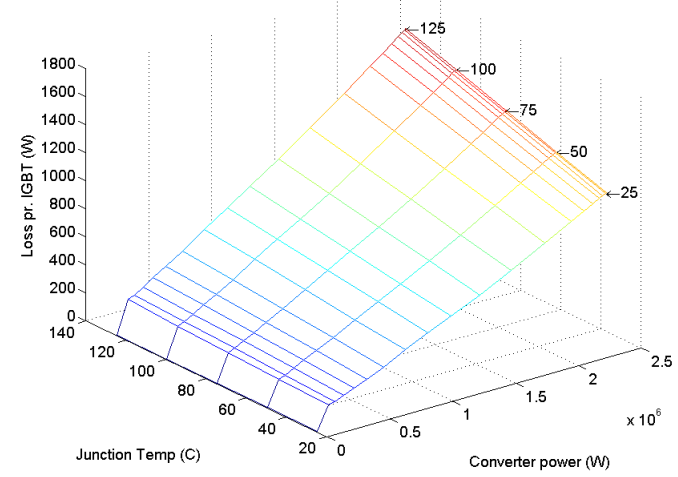

Fig. 7. 3D lookup table for the IGBT loss in the given wind power converter. 


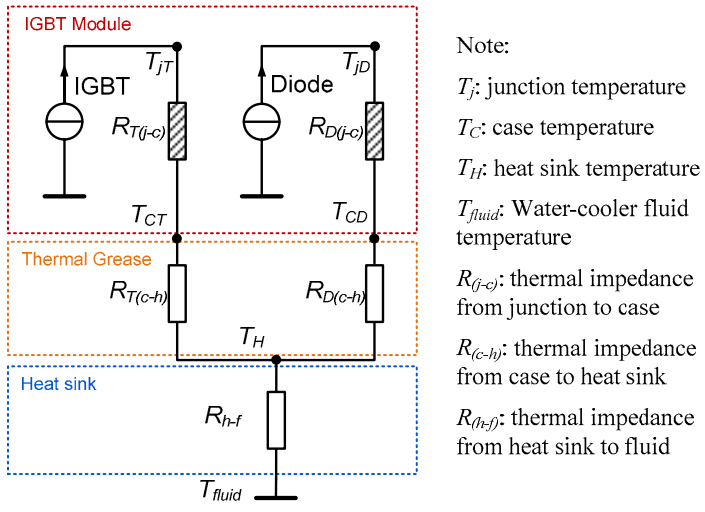

Fig. 8. Thermal network of power semiconductor devices for the long term thermal profile generation.

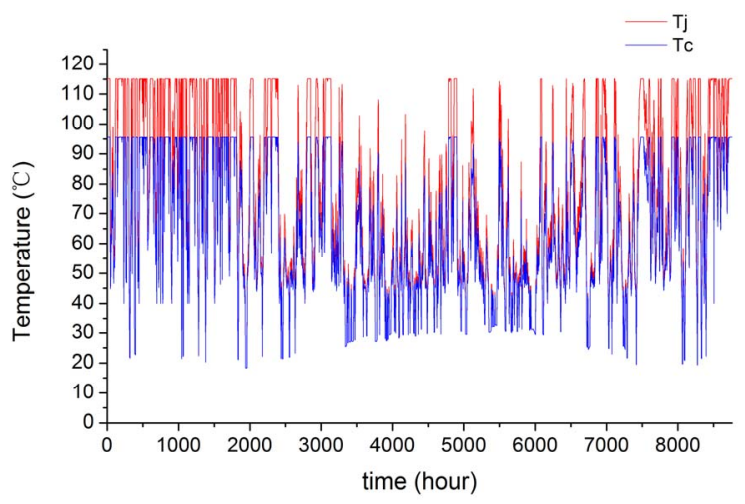

Fig. 9. One year thermal profile under the given mission profile in Fig. 3. (Junction temperature $T_{j}$ and case temperature $T_{c}$ of the IGBT, time step of 3 hour).

The thermal model (i.e. the network of thermal resistance and capacitance), which can transfer the acquired power loss in Fig. 7 to the corresponding temperature inside and outside the power devices, is an important consideration for the loading profile generation. Normally the thermal capacitance of materials will lead to fast thermal changes of the power devices range in the time constants of seconds to minutes [28] - which are still much smaller than the interested time constants for long term thermal loading. As a result, the thermal capacitance inside the power device as well as the heat sink can be ignored and only thermal resistance is taken into account for long term loading analysis. The used thermal network is shown in Fig. 8. It is noted that the fluid temperature of the water cooled heat sink $T_{\text {fluid }}$ is assumed to be maintained at $40^{\circ} \mathrm{C}$ if the IGBT is generating power losses, and $T_{\text {fluid }}$ is set to the nacelle temperature if the wind speed is below the cut in speed more than 12 hours.

Based on the above mentioned models and the 1 year mission profile shown in Fig. 3, the long term thermal loading of the IGBT modules in the given wind power converter can be generated. As shown in Fig. 9, the junction temperature $T_{j}$ of the IGBT chips, and case temperature $T_{c}$ of IGBT based plate are shown respectively because they are closely related to the major failure mechanisms of the IGBT module [16].

\section{B. Lifetime estimation with long term thermal loading}

After the long term thermal loading of the given IGBT is generated, a rain flow counting method [29], [30] has to be applied in order to convert the randomly changed thermal profile to the regulated thermal cycles which are more suitable to be utilized by the lifetime models. It is becoming a common agreement that not only the amplitude $\Delta T_{j}$ and the mean value $T_{j m}$ of thermal cycles, but also the cycling period $t_{c y c l e}$ all have strong impacts to the lifetime of power devices [15]-[18]. Different from the traditional approach, a rain flow counting method which extracts $\Delta T_{j}, T_{j m}$, and also $t_{\text {cycle }}$ is used in this paper. The counting results from the long term thermal loading in Fig. 9 is shown in Fig. 10, where 460 thermal cycles are identified and each counted cycle with its' corresponding $\Delta T_{j}, T_{j m}$, and $t_{\text {cycle }}$ are shown.

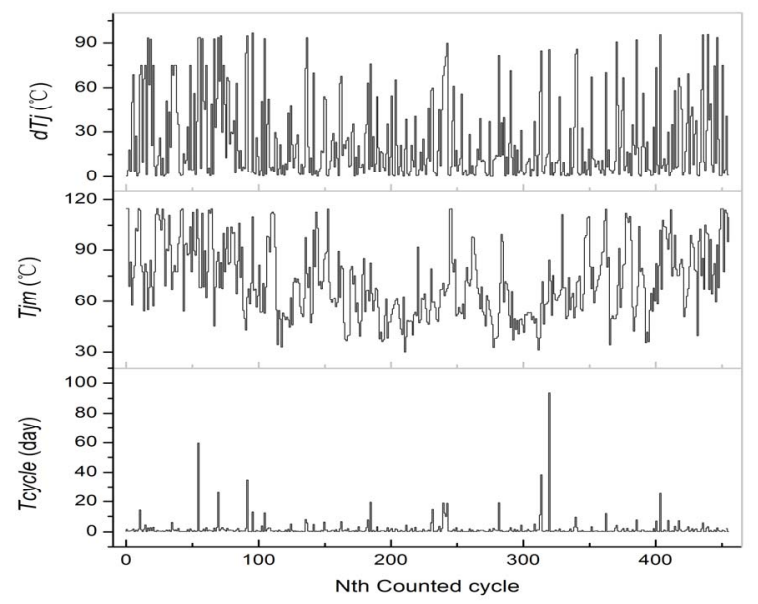

Fig. 10. Rain flow counting results of the junction temperature profile shown in Fig. 9.

After the thermal profiles are counted and regulated, the life time models for power devices can be used. There are many different approaches for lifetime modelling of power semiconductor devices, but they are not concluded yet and updated regularly [17]-[18]. Generally the life time models provided by device manufacturers are more frequently used. These life time models are based on mathematical fitting of enormous aging test data, and normally the numbers of thermal cycles $N_{\text {life }}$ to a certain failure rate $\left(B_{X}\right)$ is used as an indicator for the lifetime of power devices, which means an individual power device will have $X \%$ probability to fail (or a group of power devices have $X \%$ population to fail) after suffering $N_{\text {life }}$ of the thermal cycles.

In this paper, the life time model provided by $\mathrm{ABB}$ is used for the life time estimation [16]. This model is a series of lookup tables which can map the $n_{t h}$ counted thermal cycle in Fig. 10, to the corresponding number of cycles that the IGBT have $10 \%$ failure rate $\left(N_{n_{-} \text {life }} @ \mathrm{~B}_{10}\right)$. Then the "consumed $\mathrm{B}_{10}$ life time" by each counted thermal cycle can 


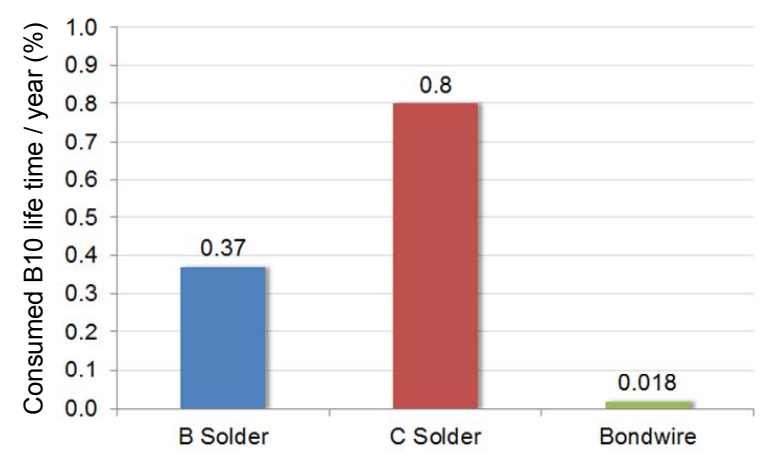

Fig. 11. Consumed $\mathrm{B}_{10}$ life time of IGBT by long term thermal cycles for one year (considering thermal cycles ranging from 3 hours to 1 year, B solder means Base plate solder, C solder means Chips solder).

be simply calculated in (1). And the total "consumed $\mathrm{B}_{10}$ lifetime" by the counted 460 long term thermal cycles in one year $C L_{\text {lyear } \text { long }}$ can be accumulated in (2) according to the Miner's rule [22].

$$
\begin{gathered}
C L_{n}=\frac{1}{N_{n_{-} \text {life }}} \\
C L_{\text {1year_long }_{\text {yel }}}=\sum_{n=1}^{460} C L_{n}
\end{gathered}
$$

The total 1 year consumed $\mathrm{B}_{10}$ life time of the IGBT module when applying the long term thermal loading in Fig. 9 are shown in Fig. 11, in which three failure mechanisms like the crack of baseplate soldering (B Solder, caused by case temperature cycling), crack of chip soldering ( $\mathrm{C}$ solder, caused by junction temperature cycling) and bond wire liftoff (bond wire, caused by junction temperature cycling) are shown respectively. It can be seen that the junction temperature cycling on the chip soldering ( $\mathrm{C}$ solder) consume more life time (i.e. more quick to failure) than the other two failure mechanisms. It is worth to mention that this life time result only reflects the influenced by long term thermal cycles with a period larger than 3 hours.

\section{MEDIUM TERM LOADING PROFILES AND LIFETIME ESTIMATION}

In order to estimate the converter life time influenced by the thermal cycles less than 3 hours, the loading profile with time constants of seconds to minutes has to be established. This group of analysis mainly focuses on the medium term thermal loading of power device caused by the mechanical behaviors of wind turbine system, such as the pitch control to limit the generated power and speed control to maximize the power production. Therefore, more complicated models and smaller time step have to be applied in order to generate enough details of the loading information and thus correctly estimate the life time. In this section the analysis is conducted with time span of 3 hours in order to restrain the data size, and a time step of 1 second is applied. However, the loading profile of 3 hours only accounts for a small part of the complete loading information in one year, which is necessary for the life time estimation of power devices. As a result, an extrapolation method from 3 hours to 1 year has to be proposed.

As shown in Fig. 12, the flow for life time estimation caused by medium term thermal loading is different from Fig. 5: the wind profile in Fig. 3 is first converted to a wind speed distribution, as indicated in Fig. 13, which represents the frequency of a certain wind speed appearing in one year $(\%$ in 365 days). Afterwards a series of wind speed variations within 3 hours are reconstructed. The used model for wind speed generation has been developed at RISØ National Laboratory based on the Kaimal spectra. The wind speed is calculated as an average value over the whole rotor, and it takes the tower shadow and the rotational turbulences into account [31]. A turbulence intensity of $18 \%$ is applied, which belong to the Class A wind turbulence [23] and is also the defined wind condition by the used V80 wind turbines. Then the rotor rotational speed and the desired average wind speed are the two inputs for the wind speed generation model. As an example shown in Fig. 15, three generated wind speed variations within 3 hours at average speed of $7 \mathrm{~m} / \mathrm{s}, 11 \mathrm{~m} / \mathrm{s}$ and $23 \mathrm{~m} / \mathrm{s}$ are illustrated.

By processing each of the reconstructed wind speed variations of 3 hours at different average values $V_{\text {ave }}$, the loading profiles generations as well as life time estimations are implemented respectively, and then the total 1 year consumed life time by medium term thermal cycles can be extrapolated by including the information of wind speed distribution. More details of the process are demonstrated as follows.

\section{A. Medium term loading profile generation}

Because the interested time constant for the medium term thermal loading is reduced to seconds' level, the dynamics of the mechanical parts cannot be ignored. In order to focus the analysis on the converter loading and lifetime estimation, an inertia transfer function with time constant of 20 seconds is added to roughly emulate the power inertia of the wind turbine, drive train and generator. Nevertheless the used models can be further detailed depending on the required accuracy.

Moreover, the turn on and turn off of power converter will introduce significant power changes and thus have strong effects on the thermal cycling of power devices, therefore the cut-in and cut-out behaviors of wind turbine should be carefully specified for life time estimation. According to the datasheet, it is defined that the rated wind speed for the used wind turbine V80 is at $12 \mathrm{~m} / \mathrm{s}$. The cut-in wind speed is set at $3 \mathrm{~m} / \mathrm{s}$ with 5 minutes average, cut out wind speed is at $25 \mathrm{~m} / \mathrm{s}$ with 5 minutes averaged or $32 \mathrm{~m} / \mathrm{s}$ with 5 seconds averaged. The re-cut in wind speed is set at $24 \mathrm{~m} / \mathrm{s}$ with a delay time of 30 minutes to emulate the startup 


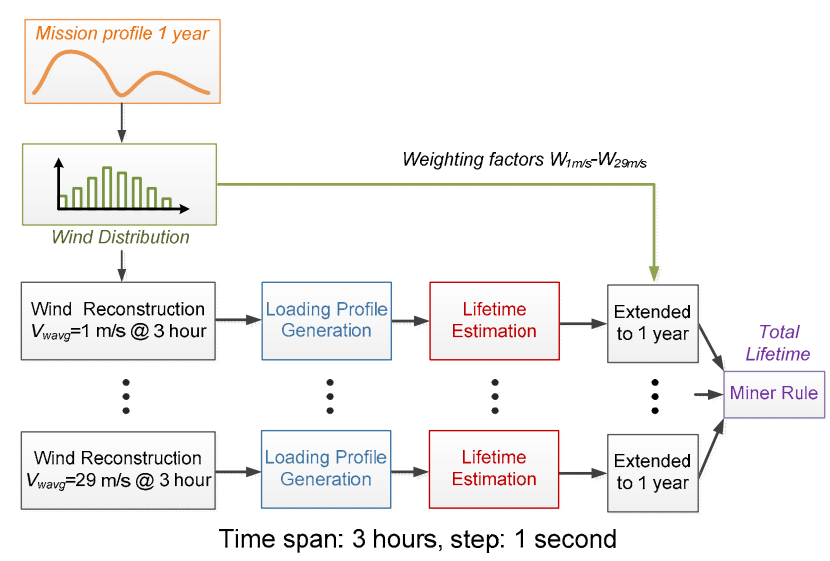

Fig. 12. Flow chart for life time estimation of power devices caused by medium term thermal cycles.

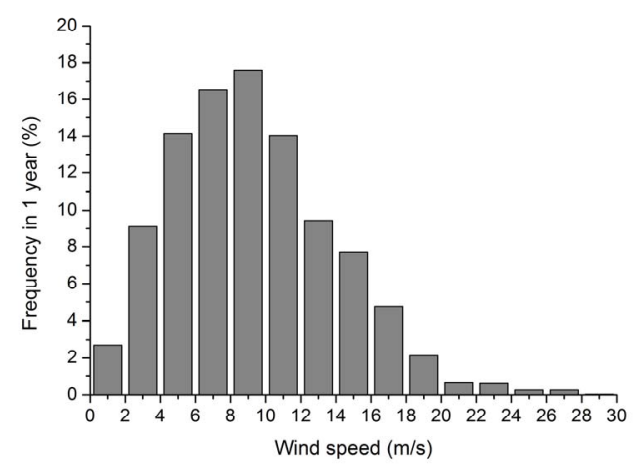

Fig. 13. Speed distribution of the given wind profile shown in Fig.3.

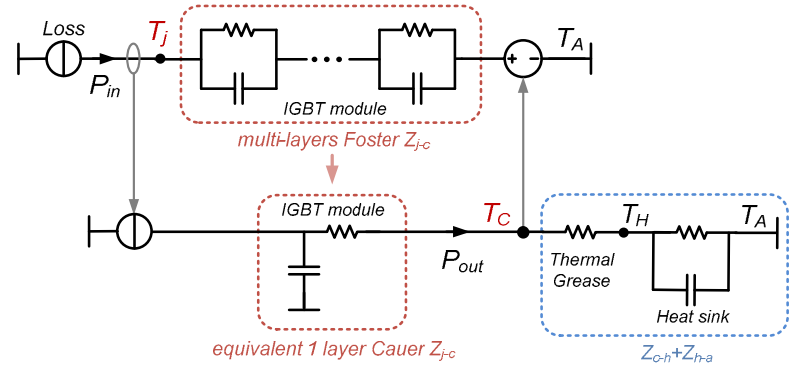

Fig. 14. Thermal network of power semiconductor devices for the medium term loading generation (only the thermal path of IGBT is illustrated for simplicity).

process of the whole wind power generation system. Based on the assumed behaviors of wind turbines, three output power of wind power converter are shown in Fig. 15, where the conditions when the average wind speed $V_{a v e}=7 \mathrm{~m} / \mathrm{s}, 11$ $\mathrm{m} / \mathrm{s}$ and $23 \mathrm{~m} / \mathrm{s}$ are illustrated respectively.

In this section, the interested thermal behaviors of power devices have much smaller time constant, therefore the thermal capacitance which determines the fast thermal dynamics ranging from seconds to minutes can no longer be ignored. Unfortunately, as detailed in [32], it is found that both of the existing Cauer and Foster thermal networks which contain thermal capacitance have their limits to acquire the appropriate case temperature of power device. As a result, a new thermal model which combines the advantages of these two thermal networks is proposed. As shown in Fig. 14, the proposed thermal model contains two paths for the thermal flow: The first one is used for junction temperature estimation and the second one is used for case and heat sink temperature estimation. By the proposed thermal model and simulation method, it is possible to estimate not only the junction temperature but also the case and heat sink temperature in a relative longer time period. When feeding the input power of converter in Fig. 15 to the loss and thermal model of devices shown in Fig. 7 and Fig. 14, the medium term thermal profile of IGBT $\left(T_{j}\right.$ and $\left.T_{c}\right)$ can be generated, as shown in Fig. 16.

\section{B. Lifetime estimation with medium term thermal loading}

With the same approach of section II to count and convert the thermal loadings in Fig. 16, the consumed lifetime of power device by medium term thermal cycles can be estimated. In order to extrapolate the life time results from 3 hour to 1 year, the thermal generation and lifetime estimation are carried out 15 times under each wind speed variation of 3 hours with different $V_{\text {ave }}$ (from $1 \mathrm{~m} / \mathrm{s}$ to $29 \mathrm{~m} / \mathrm{s}$ with step of $2 \mathrm{~m} / \mathrm{s}$ ). Then the 1 year total consumed life time

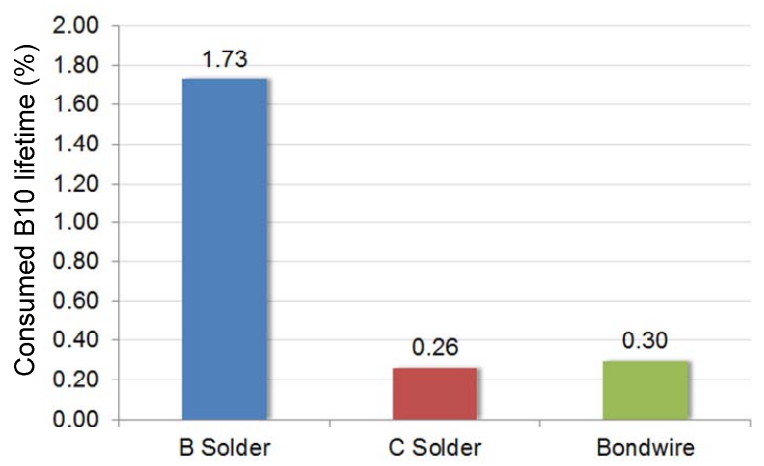

Fig. 17. Consumed $\mathrm{B}_{10}$ life time of IGBT by medium term thermal cycles for one year (three failure mechanisms, only consider thermal cycles ranging from 1 second to 3 hours, Base plate solder, C solder means Chips solder).

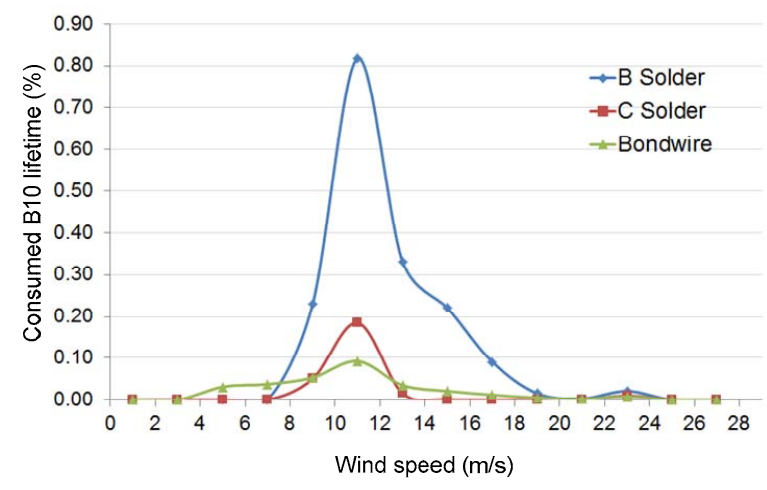

Fig. 18. Consumed $\mathrm{B}_{10}$ lifetime distribution by medium term thermal cycles (Base plate solder, C solder means Chips solder). 


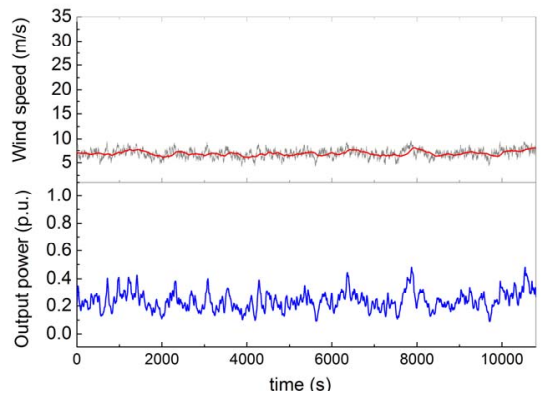

(a) $V_{\text {ave }}=7 \mathrm{~m} / \mathrm{s}$

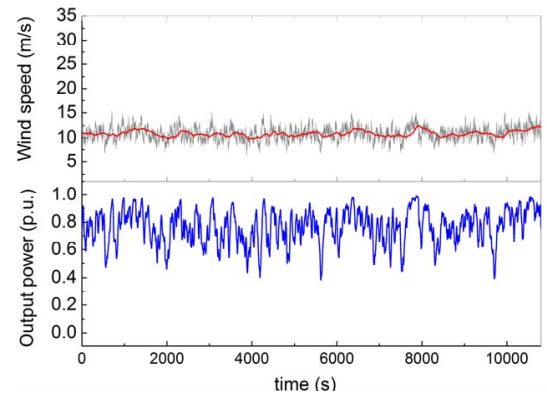

(b) $V_{\text {ave }}=11 \mathrm{~m} / \mathrm{s}$

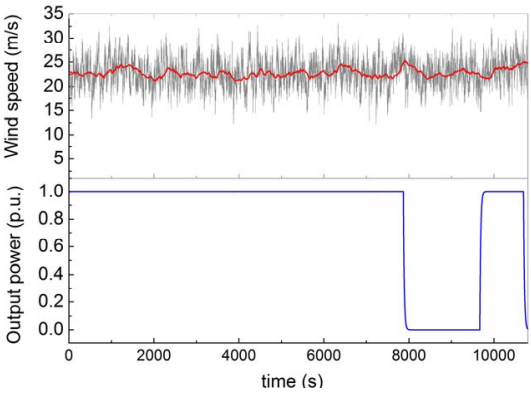

(c) $V_{\text {ave }}=23 \mathrm{~m} / \mathrm{s}$

Fig. 15. Generated wind speeds (instantaneous and 3-minute averaged) and corresponding output power of wind turbine (3 hour with 1 second time step, turbulence intensity of $18 \%$ )

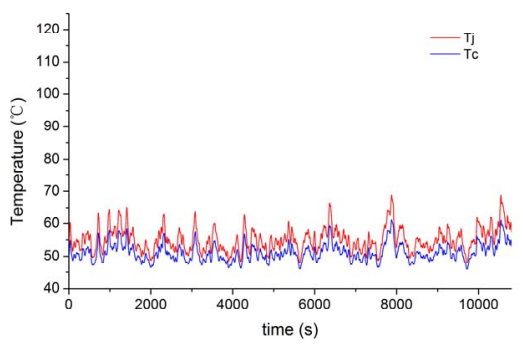

(a) $V_{\text {ave }}=7 \mathrm{~m} / \mathrm{s}$

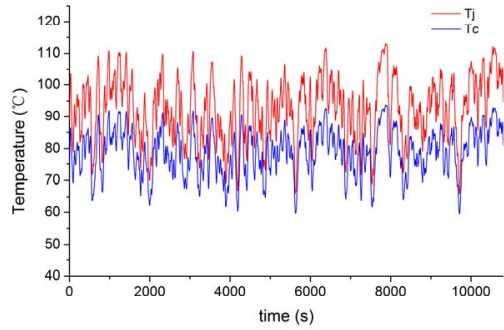

(b) $V_{\text {ave }}=11 \mathrm{~m} / \mathrm{s}$

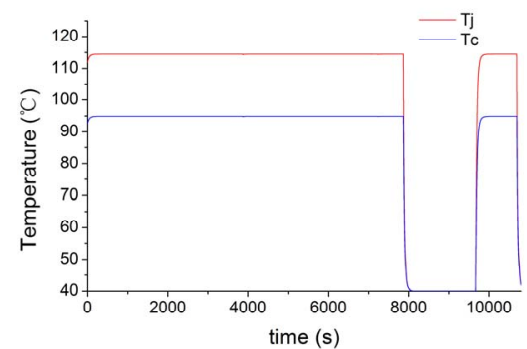

(c) $V_{\text {ave }}=23 \mathrm{~m} / \mathrm{s}$

Fig. 16. Medium term thermal loading of IGBT when applying the wind profiles in Fig. 15. (3 hour with 1 second time step).

by medium term thermal loading can be accumulated with:

$$
\begin{aligned}
& C L_{1 \text { year_medium }}=\frac{365 \cdot 24}{3}\left(W_{1 \mathrm{~m} / \mathrm{s}} \cdot C L_{1 \mathrm{~m} / \mathrm{s} \_3 \mathrm{~h}}+\right. \\
& \left.W_{3 \mathrm{~m} / \mathrm{s}} \cdot C L_{3 \mathrm{~m} / \mathrm{s} \_3 \mathrm{~h}}+\ldots+W_{29 \mathrm{~m} / \mathrm{s}} \cdot C L_{29 \mathrm{~m} / \mathrm{s} \_3 \mathrm{~h}}\right)
\end{aligned}
$$

where $W_{1 \mathrm{~m} / \mathrm{s}}$ to $W_{29 \mathrm{~m} / \mathrm{s}}$ are the weighting factors obtained from the density of wind speed distribution in Fig. 13.

The calculated 1 year consumed $\mathrm{B}_{10}$ life time of the IGBT module when applying the given medium term thermal loadings are shown in Fig. 17, in which the consumed life time by three failure mechanisms are given respectively. It is noted that this consumed life time only reflects the impacts by medium term thermal cycles with period between 1 second to 3 hours. Different from Fig. 11, the medium term case temperature cycling on the based plate of IGBT consumes more lifetime than the other two failure mechanisms.

By the proposed approach for life time estimation caused by medium term thermal loading, another interesting lifetime information can be plotted in Fig. 18 as function of wind speeds. It can be seen that the lifetime of power devices is consumed intensively at wind speeds of $10-12 \mathrm{~m} / \mathrm{s}$. This speed range is around the rated wind speed of the wind turbine, when the pitch system of wind turbine need to be activated and the output power of converter changes significantly, as can be observed in Fig. 16 (b).

\section{SHORT TERM LOADING PROFILES AND LIFETIME ESTIMATION}

In order to estimate the converter life time influenced by the short term thermal cycles less than 1 second, the loading profile with time constants of milliseconds has to be established. This group of thermal behavior is mainly caused by the fast electrical disturbances of the converter such as load current alternating, or the impacts of grid faults, etc. Therefore, small time steps are generally required for analysis.

Because it is difficult to investigate the complete loading profile of 1 year with the required small time step, the idea for life time estimation and extrapolation as shown in Fig. 12 is also applied for the lifetime analysis with short term thermal loadings. However, the methods for loading profile generation and used lifetime models are different, as detailed in the following.

\section{A. Short term loading profile generation.}

This group of thermal loading in power devices is mainly caused by the fast and periodical current alternating in the converter, the junction temperature of power devices swings at relative smaller amplitude and at constant frequency [33]. Therefore with the information of temperature mean value and cycling amplitude, the lifetime models can be directly applied without rain flow counting. It is noted that because of relative larger thermal capacitance, the thermal dynamics of the case temperature is not as fast 
as the junction temperature, thereby the case temperature are not considered for the life time estimation by short term thermal loadings.

As detailed in [34], the cycling amplitude of junction temperature $\Delta T_{j}$ caused by load current altering of converter can be analytically solved by:

$$
\Delta T_{j}=P_{\text {loss }} \cdot Z_{t h}\left(\frac{3}{8 f_{o}}\right)+2 P_{\text {loss }} \cdot Z_{t h}\left(\frac{1}{4 f_{o}}\right)
$$

where $P_{\text {loss }}$ is the loss of power device which can be looked up from Fig. 7, $Z_{t h}$ is a time-based expression of device thermal impedance, which can be found in the datasheet. $f_{o}$ is the fundamental frequency of the converter output, normally it is set at $50 \mathrm{~Hz}$ and it is also the cycling frequency of the interested thermal loadings.

In respect to the mean junction temperature, the loading profile shown in Fig. 16 can be directly used. Therefore the short term loading profile generation can be significantly accelerated because the detailed circuit models with switching behaviours can be avoided, and only an analytical function of (4), as well as a series of simulations with medium time step are needed.

\section{B. Lifetime estimation with short term thermal loading.}

It is noted that due to the lack of the testing data, most manufacturer cannot provide enough lifetime information by thermal cycles with small $\Delta T_{j}(<10 \mathrm{~K})$ and high cycling frequency $(>1 \mathrm{~Hz})$. ABB has a Coffin-Manson based life time model [35] which is tested under cycling period of 2 seconds, this is the closest testing condition that can be found and it is used in this paper for a rough approximation. The life time model can be expressed as:

$$
N_{\text {life }}=1.017^{\left(125-T_{j \mathrm{~m}}-\Delta T_{j} / 2\right)^{1.16}} \times 8.2 \cdot 10^{14} \times\left(\Delta T_{j}\right)^{-5.28}
$$

When inputting the mean junction temperature $T_{j m}$ from Fig. 16, and $\Delta T_{j}$ from (4), the consumed life time of IGBT caused by the short term thermal cycles $(50 \mathrm{~Hz})$ can be mapped. It is noted that in order to extrapolate the lifetime results from 1 second to 1 year, the simulations are carried out 15 times under 3 hours medium term wind profiles with different average speeds $(1 \mathrm{~m} / \mathrm{s}, 3 \mathrm{~m} / \mathrm{s}, 5 \mathrm{~m} / \mathrm{s} \ldots .29 \mathrm{~m} / \mathrm{s})$. Then the total consumed life time in 1 year can be accumulated according to the wind speed distribution shown in Fig. 13.

The calculated 1 year consumed life time distribution of IGBT under different wind speeds by short term thermal cycles is shown in Fig. 19. It is interesting to see that the lifetime is consumed intensively at wind speeds of 14-15 $\mathrm{m} / \mathrm{s}$, which is quite different from the lifetime distribution caused by the medium term thermal cycles in Fig. 18 (10-12 $\mathrm{m} / \mathrm{s})$. This is because the cycling amplitude of short term thermal loading is mainly decided by the absolute power loss on device (which is maximum at that wind speed), as indicated in (4), rather than the change of converter power.

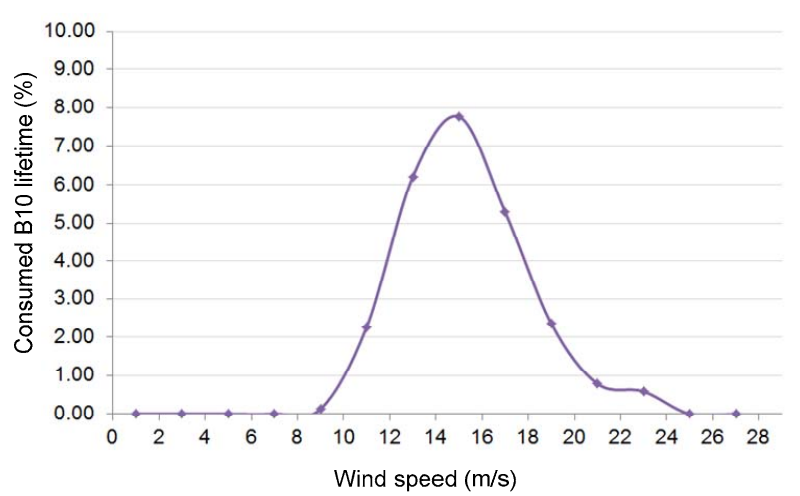

Fig. 19. Consumed $\mathrm{B}_{10}$ lifetime distribution by short term thermal cycles (only thermal cycles below 1 second).

\section{Impacts of short term thermal cycles on the life time analysis under medium term.}

The short term thermal cycles have also impacts on the amplitude of medium term thermal cycles and thus have influenced on the lifetime results with medium term thermal loadings. As indicated in Fig. 20, where the junction temperature without and with the short term thermal cycles under 3 hours medium term loading profiles $\left(V_{\text {ave }}=9 \mathrm{~m} / \mathrm{s}\right)$ are shown respectively. It can be seen that the cycling amplitude of the medium term thermal loadings increase more or less after combing the short term thermal cycles.

The rain flow counting results also agree with the

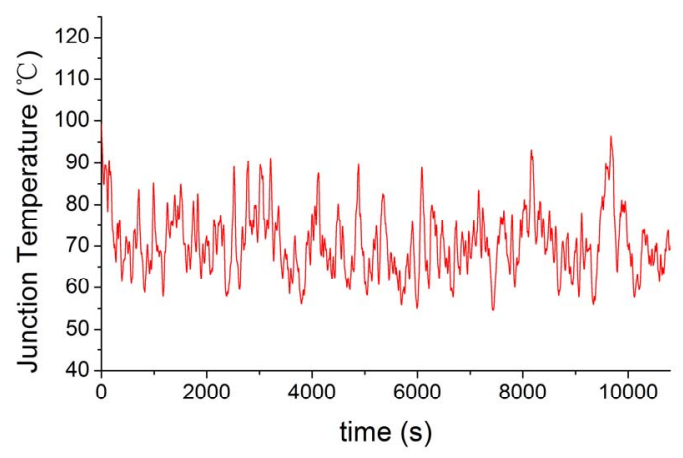

(a) Without short term thermal cycles

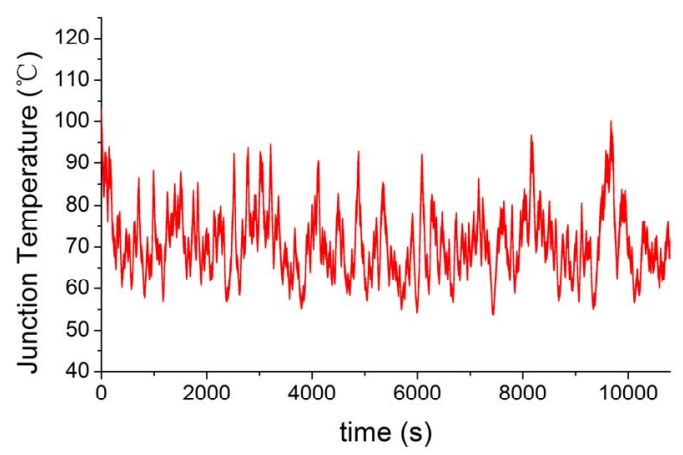

(b) Considering short term thermal cycles

Fig. 20. Medium term junction temperature of IGBT $\left(V_{\text {ave }}=9 \mathrm{~m} / \mathrm{s}, 3\right.$ hours). 
difference of these two thermal profiles. As indicated in Fig. 21 , where the rain flow counted $\Delta T_{j}$ of the two thermal profiles shown in Fig. 20 are compared. Generally the one when considering short term small thermal cycles has higher counted $\Delta T_{j}$. Fig. 22 shows the estimated 1-year consumed lifetime by this two different loading profiles. It can be seen that the short term thermal cycles do have impacts to the life time of IGBT module caused by medium term thermal loadings, especially for the failure mechanisms related to junction temperature like the chip soldering crack and the bond wire lift off.

\section{POSSIBILITIES AND LIMTS OF THE PROPOSED METHOD FOR LIFE TIME ESTIMATION}

By the proposed life time estimation approach for wind power converter, some other interesting information related to the reliability of power devices can be acquired. Fig. 23 shows the consumed life time distribution by different failure mechanisms as well as the thermal behaviors. The weak point which causes the reliability problem of the given wind power converter can be thereby discovered - Base plate and chip soldering fatigues caused by medium term thermal cycles. Because different life time models are used, the life time consumed by short term thermal cycles is not included.

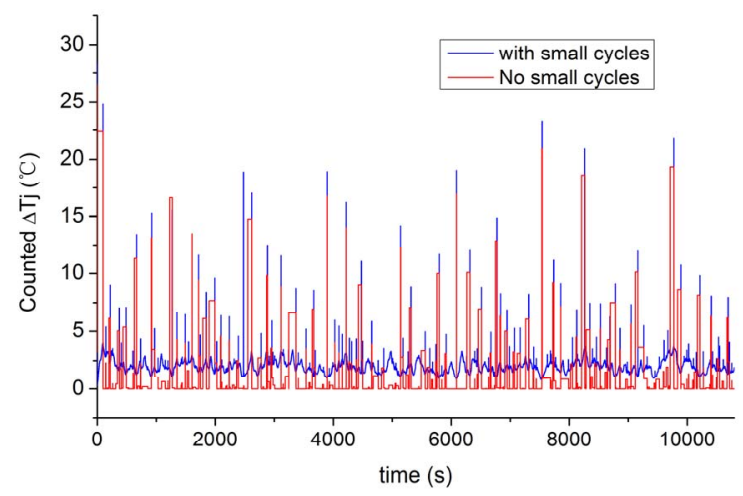

Fig. 21. Comparison of rain flow counted $\Delta T_{j}$ for the two thermal profiles shown in Fig. 20.

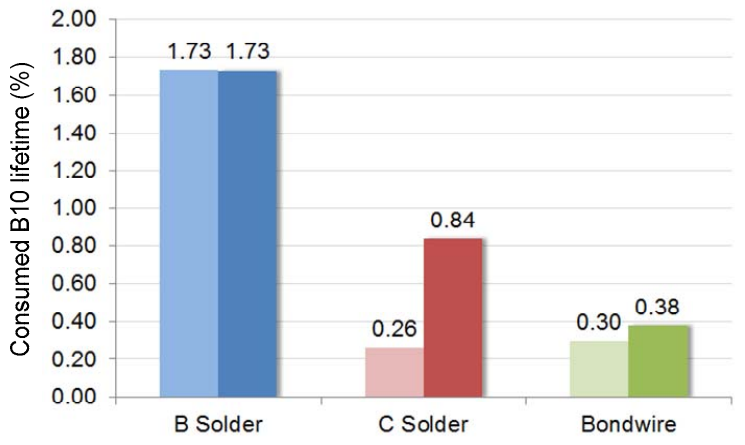

Fig. 22. Total 1-year consumed $B_{10}$ lifetime by different medium term thermal cycles (Left column: without short term thermal cycles, Right column: Considering short term thermal cycles).
Fig. 24 shows a series of consumed lifetime distribution at different wind speeds caused by medium term and short term thermal cycles, where the same loading profiles of power devices are applied with another important lifetime model from [15] and [17].

It is worth to mention that most of the lifetime models provided by manufacturer of power device are based on accelerating test results, and they can only cover very limited ranges of $\Delta T_{j}, T_{j m}$ and $t_{\text {period }}$ of the thermal cycles because the costly accelerating tests are very time consuming, and also some testing conditions are relative hard to be implemented such as very fast or very slow thermal cycling, or cycling with low mean value $T_{j m}$ but large cycling amplitude $\Delta T_{j}$, etc. However, it is found that in the wind power applications many loading conditions of the power devices which are not yet tested to map the lifetime information could be presented. In order to translate these "unidentified thermal cycles" into lifetime, log linear method are used to interpolate and extrapolate the existing life time models - this could lead to some in-confidence of the acquired lifetime results. Therefore maybe even more advanced lifetime models which can cover more loading conditions of power devices are required for the mission profile-based lifetime estimation of power electronics

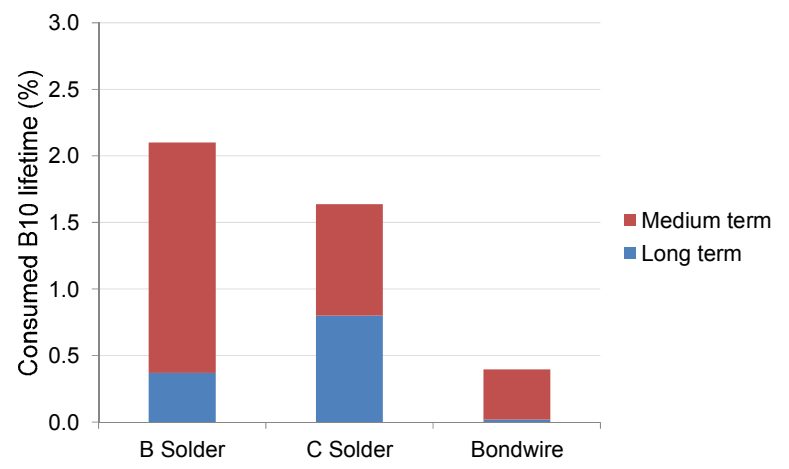

Fig. 23. Total 1-year consumed life time distribution by different failure mechanisms and thermal behaviors (short term thermal cycles are considered in medium term thermal loading).

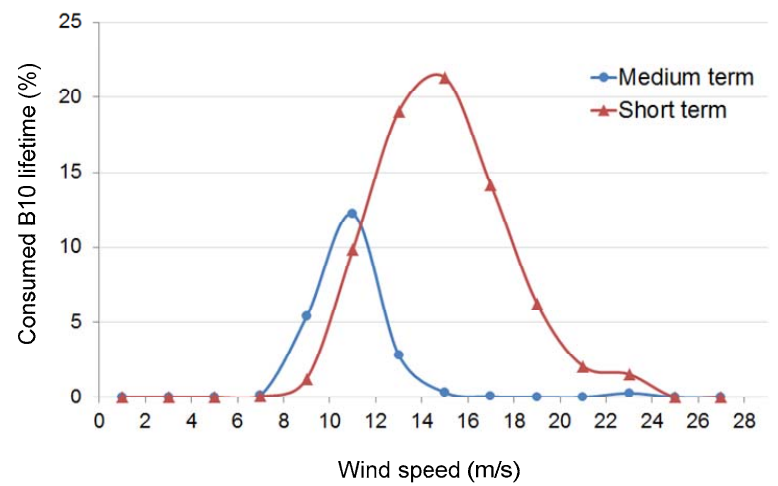

Fig. 24. Consumed lifetime distribution of IGBT by medium and short term thermal cycles (life time model from [15], [17]). 
converter.

\section{CONCLUSIONS}

A lifetime estimation approach for wind power converter is proposed in this paper. It is based on the loading and strength analysis of power devices and separates the analysis under different time constants of the thermal behaviors in wind turbine system. With the established methods for loading profile generation and life time estimation, more possibilities and details of the lifetime information for wind power converter like the distribution of lifetime consumption by different thermal behaviors, wind speeds, and failure mechanisms can be obtained. This is very useful to indicate and improve the weakness of the system in respect to the reliability performance.

It is also found that in the wind power application many loading conditions of power devices which are not covered by most of the lifetime models could be presented, therefore more advanced lifetime modeling are required for the mission profile-based lifetime estimation of power converter.

\section{REFERENCES}

[1] F. Blaabjerg, M. Liserre, K. Ma, "Power Electronics Converters for Wind Turbine Systems," IEEE Trans. on Industrial Application, vol. 48, no. 2, pp. 708-719, 2012.

[2] M. Liserre, R. Cardenas, M. Molinas, J. Rodriguez, "Overview of Multi-MW wind turbines and wind parks", IEEE Trans. on Industrial Electronics, Vol. 58, No. 4, pp. 1081-1095, April 2011.

[3] Z. Chen, J.M. Guerrero, F. Blaabjerg, "A Review of the State of the Art of Power Electronics for Wind Turbines," IEEE Trans. on Power Electronics, vol.24, No.8, pp.1859-1875, Aug 2009.

[4] S. Faulstich, P. Lyding, B. Hahn, P. Tavner "Reliability of offshore turbines-identifying the risk by onshore experience," in Proc. of European Offshore Wind, Stockholm, 2009.

[5] B. Hahn, M. Durstewitz, K. Rohrig "Reliability of wind turbines - Experience of 15 years with 1500 WTs", Wind Energy, Spinger, Berlin, 2007.

[6] Wikipedia "Cost of electricity by source," April 2013.

[7] Report of the US Energy Information Administration (EIA) of the U.S. Department of Energy (DOE). "Levelized Cost of New Generation Resources in the Annual Energy Outlook 2013," Released in spring, 2013.

[8] ZVEL, Handbook for robustness validation of automotive electrical/electronic modules, Jun. 2008. (Available: http://www.zvei.org/)

[9] E. Wolfgang, L. Amigues, N. Seliger and G. Lugert, "Building-in Reliability into Power Electronics Systems". The World of Electronic Packaging and System Integration, pp. 246-252, 2005.

[10] D. Hirschmann, D. Tissen, S. Schroder, R.W. De Doncker, "Inverter design for hybrid electrical vehicles considering mission profiles," IEEE Conference on Vehicle Power and Propulsion, pp. 1-6, 2005.

[11] C. Busca, R. Teodorescu, F. Blaabjerg, S. Munk-Nielsen, L. Helle, T. Abeyasekera, P. Rodriguez, "An overview of the reliability prediction related aspects of high power IGBTs in wind power applications," Microelectronics Reliability, Vol. 51, no. 9-11, pp. 1903-1907, 2011.
[12] E. Wolfgang, "Examples for failures in power electronics systems," presented at ECPE Tutorial on Reliability of Power Electronic Systems, Nuremberg, Germany, April 2007.

[13] S. Yang, A. T. Bryant, P. A. Mawby, D. Xiang, L. Ran, and P. Tavner, "An industry-based survey of reliability in power electronic converters," IEEE Trans. on Ind. Appl., vol. 47, no. 3, pp. 1441- 1451, May/Jun. 2011.

[14] J. Due, S. Munk-Nielsen, Rasmus Nielsen, "Lifetime investigation of high power IGBT modules", in Proc. of EPE'2011, pp. 1-8, 2011.

[15] A. Wintrich, U. Nicolai, T. Reimann, "Semikron Application Manual," pp. 128, 2011

[16] J. Berner, "Load-cycling capability of HiPak IGBT modules,' ABB Application Note 5SYA 2043-02, 2012.

[17] U. Scheuermann, "Reliability challenges of automotive power electronics," Microelectronics Reliability, vol. 49, no. 9-11, pp. 1319-1325, 2009.

[18] U. Scheuermann, R. Schmidt, "A New Lifetime Model for Advanced Power Modules with Sintered Chips and Optimized Al Wire Bonds," Proc. of PCIM' 2013, pp. 810-813, 2013.

[19] H. Wang, K. Ma, F. Blaabjerg, "Design for reliability of power electronic systems," Proc. of IECON' 2012, pp. 33-44, 2012.

[20] H. Wang, D. Zhou, "A Reliability-Oriented Design Method for Power Electronic Converters," Proc. of APEC' 2013, pp. 2921-2928, 2013.

[21] O. S. Senturk, S. Munk-Nielsen, R. Teodorescu, L. Helle, P. Rodriguez, "Electro-thermal modeling for junction temperature cycling-based lifetime prediction of a press-pack IGBT 3L-NPC-VSC applied to large wind turbines", Proc. of ECCE'11, pp. 568-575, 2011

[22] M. A. Miner, "Cumulative damage in fatigue," Journal of Applied Mechanics, no. 12, A159-A164, 1945.

[23] Wikipedia "IEC 61400," June 2013.

[24] Website of Vestas Wind Power, Wind turbines classes, June 2013. (Available: http://www.vestas.com/).

[25] Website of Vestas Wind Power, Wind turbines overview, June 2013. (Available: http://www.vestas.com/).

[26] User manual of PLECS blockset version 3.1, March 2011.

[27] D. Graovac, M. Purschel, "IGBT Power Losses Calculation Using the Data-Sheet Parameters," Infineon Application Note, January, 2009.

[28] Infineon Application Note AN2008-03: "Thermal equivalent circuit models", June 2008.

[29] ASTM International, E1049-85 (2005) Standard practices for cycle counting in fatigue analysis, 2005.

[30] A. NIESŁONY, "Determination of fragments of multiaxial service loading strongly influencing the fatigue of machine components," Mechanical Systems and Signal Processing, Vol. 23, No.8, pp. 2712-2721, 2009.

[31] P. Sørensen, A. D. Hansen, P.A.C. Rosas, "Wind models for simulation of power fluctuations from wind farms," Journal of Wind Engineering, vol. 90, 2002, pp. 1381-1402.

[32] K. Ma, F. Blaabjerg, M. Liserre, "Electro-thermal model of power semiconductors dedicated for both case and junction temperature estimation," Proc. of PCIM' 2013, pp. 1042-1046, 2013.

[33] K. Ma, F. Blaabjerg, "Multilevel Converters for 10 MW Wind Turbines," in Proc. of EPE' 2011, pp. 1-8, August 2011.

[34] K. Ma, F. Blaabjerg, "Reliability-Cost Models for the Power Switching Devices of Wind Power Converters," Proc. of PEDG' 2012, pp.820-827, 2012.

[35] N. Kaminsku, "Load-cycling capability of HiPak," ABB Application Note 5SYA 2043-01, 2004. 